\title{
Dashboard Visualization of Information for Emergency Medical Services
}

\author{
Oliver M. CHRISTEN ${ }^{\mathrm{a}, 1}$, Yannic MÖSCHING ${ }^{\mathrm{a}, 1}$, Patrik MÜLLER $^{\mathrm{b}}$, \\ Kerstin DENECKE ${ }^{\mathrm{a}, 2}$ and Stephan NÜSSLI ${ }^{\mathrm{a}}$ \\ a Bern University of Applied Sciences, Biel, Switzerland \\ ${ }^{\mathrm{b}}$ Emmental Hospital, Burgdorf, Switzerland
}

\begin{abstract}
In emergency situations, every minute counts. Therefore, staff of emergency medical services (EMS) require easily accessible sources of information to organize and coordinate their work as quickly as possible. Digital dashboards can visualize various information at a glance and have thus potential to meet this need. We developed in cooperation with the Emmental Hospital a prototype of a dashboard, which aims to improve organizational aspects of the EMS. Method: A literature search was conducted in PubMed, IEEE and ACM. The goal was to identify design principles for dashboards. Additionally, several interviews and meetings were held with the EMS staff of the Emmental Hospital and with those of another hospital. The aim was to identify requirements of the EMS staff towards such an organizational dashboard and to transform them into use cases. Results: Considering the collected requirements and standards of dashboard design, a prototype of a dashboard was developed. It consists of several modules that show relevant information items such as news or traffic information. Due to this modular development, content is easily interchangeable. The most important information for the EMS is shown on the dashboard aiming at saving time for information gathering. Conclusion: A digital dashboard offers many advantages and optimization possibilities compared to an analog whiteboard. For example, such a dashboard can be connected to other systems and data can be automatically included. Although we developed our dashboard in cooperation with the EMS of a specific hospital, it can easily be applied and adjusted to other EMS. As a next step, we will perform usability tests with the prototype and start implementing the dashboard.
\end{abstract}

Keywords. Emergency medical service, information system, visualization, dashboard

\section{Introduction}

In medical emergency situations every minute counts; as soon as an emergency alert arrives paramedics need to move out immediately. Therefore, they are in need to easily access information in order to organize and coordinate their work as fast as possible. Dashboards show relevant information at a glance and have thus potential to meet this need. More specifically, dashboards generally display the most important data needed to achieve a certain objective [1]. In the case of a dashboard for emergency medical services

${ }^{1}$ Contributed equally and share first authorship

${ }^{2}$ Corresponding Author, Kerstin Denecke, Bern University of Applied Sciences, Institute for Medical Informatics, Quellgasse 21,2502 Biel, Switzerland; E-mail: kerstin.denecke@bfh.ch 
(EMS) the objective is to display all the relevant information that the team members need to efficiently organize themselves.

Dashboards have already proven to be helpful in displaying information needed in critical situations [2] or for supporting decision making. Reis et al. [3] developed a dashboard prototype to keep track of patients arriving at the hospital emergency service. Patients were given a bracelet which monitored their vital signs and sent the acquired data to a database. The dashboard displayed the data from the database to help health professionals in keeping track of patients' condition. Pestana et al. [4] developed dashboards to improve health care management in hospitals. They designed and evaluated dashboards to display key performance indicators of hospital departments. In this context, dashboards served as a decision support tool for the hospital management.

In the EMS of our cooperation partner Emmental Hospital a dashboard is already in use implemented as a whiteboard that organizes part of their information sources. The whiteboard displays information about the team and the vehicle department, as well as absences and shift information regarding team members. Team leaders as well as team members are responsible for keeping the displayed data up to date. Aside from the mentioned whiteboard, the team members use a file share to access information about traffic messages and internal directives. This requires that team members regularly log into their computers or tablets to check for updates, which is time consuming. The goal of this work is to develop a technical tool that consolidates the available information sources, summarizes the relevant data and present it in one central display, which is the dashboard. In this way, we intend to facilitate the work of the EMS team members, so that they do not have to collect their needed information from multiple sources and thus can save time.

\section{Methods}

A literature search was conducted between February and June 2020 in the online databases PubMed, IEEE and ACM. The purpose of the literature search was to extract principles in dashboard design and evaluate standards, which we wanted to consider in the prototype development. Further, we wanted to find out whether such or similar solutions already exist on the market and whether positive results can be achieved with them. The terms and keywords for the search were (dashboard OR tablet application) AND (design OR evaluation), dashboard AND (emergency OR ambulance OR paramedic), dashboard AND (ambulance OR emergency services OR emergency medical services OR paramedic), located either within the abstract or listed as keywords. The search resulted in the following results: IEEE (331 results), Pubmed (238 results), ACM (284 results). Based on the title, irrelevant papers were filtered out by checking the title and reading the abstract. This process returned 22 relevant paper where the full text was read. Finally, 4 papers were considered relevant for our work. Papers have been excluded when they were inconsistent with our core topics. In this way, we removed publications that did not focus on dashboards, the management of EMS or the design of dashboards. The full texts of all remaining publications were read.

In order to identify the requirements, five interviews and meetings were conducted with the EMS staff of the Emmental Hospital and with those of another hospital. These interviews were conducted on a small scale with a maximum of four persons. Among 
them were an IT specialist, EMS employees, but also a team leader who is taking care of administrative issues within the EMS

We thus gained insights into the organization of EMS and revealed the information systems and structures that are currently in place. Furthermore, ideas and suggestions for information visualization were collected. The collected requirements were evaluated, prioritized and divided into functional and non-functional requirements. All requirements were described as use cases and the use cases were formalized in a use case diagram. Based on this diagram as well as the results of the literature search on dashboard design, a prototype was developed using Axure RP 9.

\section{Results}

In this section the collected requirements and standards for dashboard visualization are summarized and our prototype is described.

\subsection{Requirements and use case descriptions}

Traffic messages: The EMS currently receives information about the exact traffic situation such as road closures or traffic restrictions. These messages are received as Word or PDF file. On the dashboard these messages should be visible at a glance.

Short news: To receive directives and general information, currently the platform Smedex (http://smedex.com) is used by the staff of the EMS. Smedex is an e-learning platform on which mandatory courses and educational certificates for the continuous education can be managed. When new entries are made in Smedex, the users of the dashboard should be informed. This functionality requires an interface to Smedex.

Allocation of staff and vehicles: The allocation of staff and vehicles is done in a different system. This information should be taken from the system and clearly displayed on the dashboard.

Weekly vehicle control (functional requirement): The ambulance vehicles are thoroughly checked according to a flexible checklist. Materials are replaced and maintenance is carried out if necessary. The checklist should be displayed on the dashboard.

EMS staff attendances and absences: Attendances and absences are currently recorded in a different system. The dashboard should provide an interface to this system so that staff attendances and absences are displayed directly on the dashboard.

External material: Material that was stored during emergency deployments in other hospitals and is to be returned should be displayed on the dashboard.

Medication expiration dates: Muscle relaxants are stored in the emergency vehicles and expire within a short time. The expiration dates should be visible and editable on the dashboard.

Usability (non-functional requirement): The dashboard is intended to facilitate and simplify the coordination of the EMS. Therefore, the design of the dashboard has to ensure high usability. This comprises that all relevant information should be visible at first glance or after one click at most. Further, adapting information should be as easy as possible.

Display of the dashboard (non-functional requirement): The dashboard should be displayed on mobile devices as well as on large screens. 


\subsection{Dashboard Design}

The following definition states the main purpose of dashboards: "A dashboard is a visual display of the most important information needed to achieve one or more objectives" [1]. We could not identify any fixed criteria that determine the process or appearance of dashboard designs. Nevertheless, we collected some points that should be considered in order to optimize the presentation. A dashboard should only display as much data as necessary to meet the users' objectives [5]. Users want to see the most important information at a glance. If a dashboard provides a large amount of information, important data might get lost in the crowd. If the same measurements are presented, these data should be presented in a consistent manner, e.g. using the same measurement unit. Graphs, numbers or other elements should always be displayed in the same way. Consistency is not only important in the choice of data visualization, but should also be applied to other points such as font or layout. Some key figures on a dashboard are closely interwoven. The information content is rather higher when all values can be viewed simultaneously than when the key figures have to be perceived one after the other. On a well-designed dashboard, related metrics are grouped close together $[1,6]$.

\subsection{Prototype}

The resulting prototype can be seen in figure 1. The dashboard visualizes information from the individual requirements. For example, the traffic messages are displayed in the upper left corner and the allocation of staff and vehicles are displayed to the right. Much of the layout was inherited from the existing whiteboard to keep some consistency for the users. However, since new information was added, the order of the information pieces was modified.

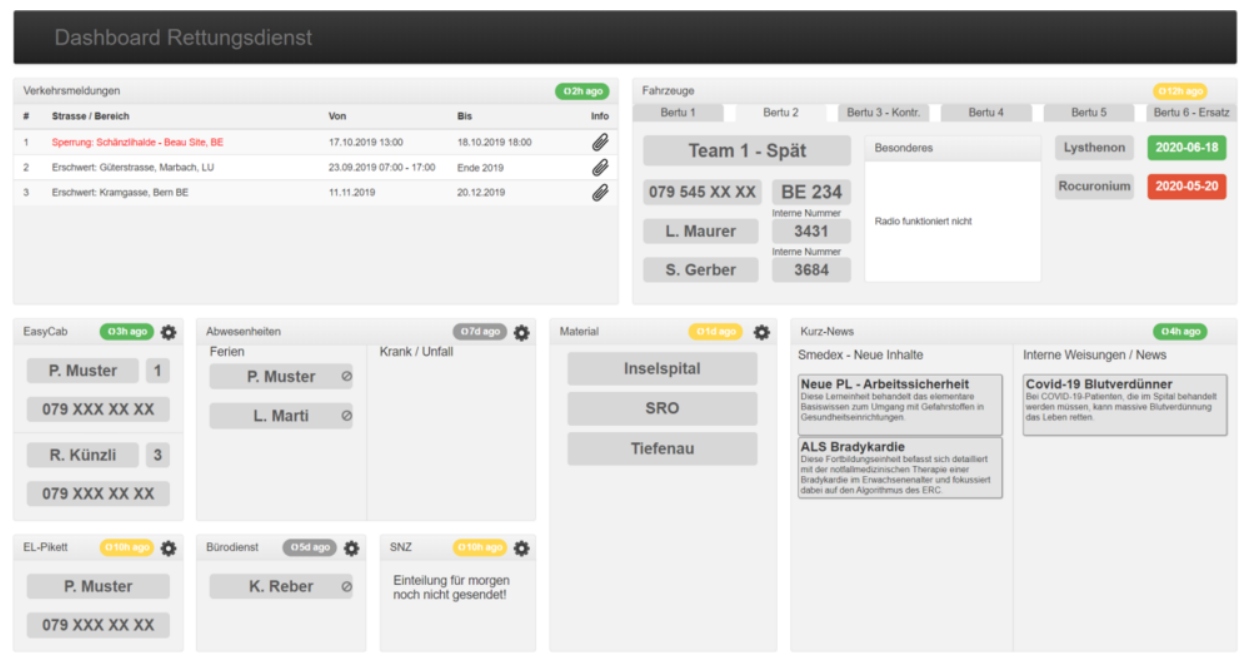

Figure 1. Dashboard prototype

The requirement "Traffic messages" was met by using a table to display where and for how long traffic is blocked due to construction. For every entry in the table a symbolic link can be opened to display the corresponding document, which contains further 
information. The requirement "Short news" was met by listing new entries from Smedex and internal notifications next to each other.

Panels with borders visually separate the different parts of the dashboard from each other to allow for a clear layout. For every panel a small label indicates when the respective panel was last updated. These labels are supposed to help viewers, to recognize parts of the dashboard that have changed recently.

\section{Discussion and Conclusions}

Several software solutions for EMS are available on the market. We identified two commercial solutions that met partially our collected requirements. Both products offer comprehensive solutions, but do not meet all requirements of our cooperation partner. Therefore, we decided to develop a prototype specifically tailored to the collected requirements to achieve an optimal solution.

The requirement analysis has shown that a digital dashboard offers many advantages and optimization possibilities compared to an analog whiteboard. For example, the dashboard can be connected to other systems via interfaces. Thus, data can be automatically transferred to the dashboard and displayed. In this way, the actuality of the displayed information can be ensured. Machine learning technologies can be used to identify and prioritize favorites of information - for example, in case of lists, the most frequently mentioned objects would be displayed at the top. Additionally, access to an external file share is no longer required which can save time. All data can be accessed via the dashboard. Colors or other visual features can be used to draw the attention of viewers to important information. A digital dashboard can also be displayed on tablets so users always have access to it when they are on the way to an intervention.

The described dashboard is based on the requirements of the Emmental Hospital EMS. By interviewing staff of another EMS, we tried to ensure that our solution is not only addressing the needs of one hospital. Additionally, we developed our dashboard in a modular way. In this way, we can ensure that it can be adopted and adjusted for the EMS of another hospital. We assume that some small individual adjustments would be necessary when deploying our dashboard to another EMS.

As a next step, we will conduct a usability test with the prototype. Afterwards, the necessary improvements will be integrated, and the dashboard will be implemented.

\section{References}

[1] Few S. Information dashboard design: The effective visual communication of data. Beijing, London: OReilly; 2006.

[2] Nascimento BS, Vivacqua AS, Borges MRS. A flexible architecture for selection and visualization of information in emergency situations. 2016 IEEE International Conference on Systems, Man, and Cybernetics (SMC), pp. 3317-3322.

[3] Reis A, Coutinho F, Ferreira J, et al. Monitoring System for Emergency Service in a Hospital Environment. 2019 IEEE $6^{\text {th }}$ Portuguese Meeting on Bioengineering (ENBENG), pp. 1-4.

[4] Pestana M, Pereira R, Moro S. Improving Health Care Management in Hospitals Through a Productivity Dashboard. Journal of Medical Systems 44 (4), p. 87.

[5] Sarikaya A, Correll M, Bartram L, Tory M, Fisher D. What Do We Talk About When We Talk About Dashboards? IEEE Transactions on Visualization and Computer Graphics 2018; 25(1):682-92.

[6] Effective dashboard design: a step-by-step guide | Geckoboard; 2020 [From: 10.05.2020]. Available on: https://www.geckoboard.com/best-practice/dashboard-design/. 